\title{
GLOBALIZATION OF SOME LOCAL PROPERTIES IN KRULL DOMAINS
}

\author{
D. D. ANDERSON
}

\begin{abstract}
Let $R$ be a Krull domain. It is shown that a nonzero locally principal ideal is invertible. This is used to show that $\mathrm{Cl}(R) / \mathrm{Pic}(R)$ is torsion if and only if $\mathrm{Cl}\left(R_{M}\right)$ is torsion for each maximal ideal $M$ of $R$. Here $\mathrm{Cl}(R)$ and $\operatorname{Pic}(R)$ denote the divisor class group and Picard group of $R$, respectively.
\end{abstract}

1. Introduction. Perhaps the most interesting result of this note is that a nonzero locally principal ideal in a Krull domain is invertible. This result is a special case of the following theorem we prove in $\S 2$ : Let $I$ be a finite type $v$-ideal in an integral domain $R$ with the property that for each maximal ideal $M$ of $R,\left(I^{n}\right)_{v M}$ is principal for some positive integer $n$ (depending on $M$ ). Then $\left(I^{N}\right)_{v}$ is invertible for some positive integer $N$. In $\S 3$ the results of $\S 2$ are used to globalize some local properties in Krull domains. We give many conditions equivalent to a Krull domain $R$ having $\mathrm{Cl}\left(R_{M}\right)=0$ (i.e., $R$ is locally factorial), or $\mathrm{Cl}\left(R_{M}\right)$ being torsion (i.e., $R$ is locally almost factorial) for every maximal ideal $M$ of $R$. The main result of $\S 3$ is that $\mathrm{Cl}(R) / \mathrm{Pic}(R)$ is torsion if and only if $\mathrm{Cl}\left(R_{M}\right)$ is torsion for each maximal ideal $M$ of $R$, where $R$ is a Krull domain. Here, as usual, $\mathrm{Cl}(R)$ and $\mathrm{Pic}(R)$ denote the divisor class group and Picard group of $R$, respectively.

2. Locally principal ideals. Let $R$ be an integral domain with quotient field $K$. For a nonzero ideal $I$ of $R$, we write $I_{v}$ for $\left(I^{-1}\right)^{-1}$ and say that $I$ is a divisorial ideal or a $v$-ideal if $I=I_{v}$. We say that $I$ is a finite type $v$-ideal if $I=J_{v}$ for some finitely generated ideal $J$ of $R$. It is well known that every divisorial ideal of a Krull domain is of finite type. An ideal $I \neq 0$ is called a $t$-ideal if for nonzero $x_{1}, \ldots, x_{n} \in I,\left(x_{1}, \ldots, x_{n}\right)_{v} \subseteq I$. Thus $I$ is a $t$-ideal if and only if $I=\bigcup J_{v}$ where $J$ runs over the set of nonzero finitely generated ideals of $R$ contained in $I$. Evidently a finite type $t$-ideal is a (finite type) $v$-ideal. Let $\operatorname{Max}(R)$ be the set of maximal ideals of $R$. An ideal $I$ is locally principal if $I_{M}$ is principal for each $M \in \operatorname{Max}(R)$. It is well known that a nonzero ideal $I$ is invertible if and only if it is finitely generated and locally principal. Our first result weakens the requirement that $I$ be finitely generated. Our general references are [5] and [6].

THEOREM 2.1. Let I be a nonzero locally principal ideal in an integral domain. Then $I$ is a t-ideal. Further, $I$ is invertible if and only if $I$ has finite type.

Proof. Let $x_{1}, \ldots, x_{n} \in I$. Then for $t \in\left(x_{1}, \ldots, x_{n}\right)_{v}=R:\left(R:\left(x_{1}, \ldots, x_{n}\right)\right)$, $t\left(R:\left(x_{1}, \ldots, x_{n}\right)\right) \subseteq R$. Let $M \in \operatorname{Max}(R)$. Hence

$$
t\left(R_{M}:\left(x_{1}, \ldots, x_{n}\right)_{M}\right)=t\left(R:\left(x_{1}, \ldots, x_{n}\right)\right)_{M} \subseteq R_{M}
$$

Received by the editors May 27, 1981.

1980 Mathematics Subject Classification. Primary 13F15, 13 C13.

Key words and phrases. Krull domain, locally factorial, divisor class group.

(C) 1982 American Mathematical Society 0002-9939/81/0000-1057/\$02.25 
that is, $\left(x_{1}, \ldots, x_{n}\right)_{v} \subseteq\left(\left(x_{1}, \ldots, x_{n}\right)_{M}\right)_{v} \subseteq I_{M}$ because $I_{M}$ is principal and hence divisorial. Since this is true for all $M \in \operatorname{Max}(R)$, we have $\left(x_{1}, \ldots, x_{n}\right)_{v} \subseteq I$. Thus $I$ is a $t$-ideal.

If $I$ is invertible, then $I$ is even finitely generated. Conversely, suppose that $I$ is locally principal and that $I=J_{v}$, where $J$ is a finitely generated ideal. Let $0 \neq b \in I$. We show that $(b)=I((b): I)$ from which it follows that $I$ is a factor of a principal ideal and therefore invertible. First, note that $(b): I=(b): J_{v}=(b): J$. Let $M$ be a maximal ideal of $R$. Then $(b)_{M} \subseteq I_{M}$ and $I_{M}$ is principal, so we have $(b)_{M}=I_{M}\left((b)_{M}: I_{M}\right)$. However,

$$
((b): I)_{M}=((b): J)_{M}=(b)_{M}: J_{M} \supseteq(b)_{M}: I_{M} \supseteq((b): I)_{M} \text {. }
$$

Hence $((b): I)_{M}=(b)_{M}: I_{M}$. (Here we have used the fact that $J$ is finitely generated to get $((b): J)_{M}=\left((b)_{M}: J_{M}\right)$. Thus

$$
(b)_{M}=I_{M}\left((b)_{M}: I_{M}\right)=I_{M}((b): I)_{M}=(I((b): I))_{M} \text {. }
$$

Since this is true for all $M \in \operatorname{Max}(R)$, we have that $(b)=I((b): I)$.

The proof of Theorem 2.1 actually shows that any locally $t$-ideal is a $t$-ideal. In particular, if $R$ is a Krull domain, an ideal is divisorial if and only if it is locally divisorial. This and the next corollary follow from the previously mentioned result that every $v$-ideal in a Krull domain is of finite type.

COROLlaRY 2.2. A nonzero ideal in a Krull domain is invertible if and only if it is locally principal.

In [6, p. 519] an example of an almost Dedekind domain $D$ is given in which every maximal ideal but one, say $M$, is invertible. It is shown that $M^{-1}=D$. Thus $M$ is locally principal (and hence a $t$-ideal), but $M$ is not a $v$-ideal.

We introduce the following notation to simplify the statement of our next theorem. For a nonzero ideal $I$ of $R$, we define $I^{(n)}=\left(I^{n}\right)_{v}$. Thus $I^{(1)}=I_{v}$. If $I^{(1)}$ has finite type, say $I_{v}=J_{v}$, where $J$ is finitely generated, then $I^{(n)}=\left(I^{n}\right)_{v}=\left(J^{n}\right)_{v}$, so that $I^{(n)}$ also is of finite type. Finally, note that $I^{(n)(l)}=I^{(n l)}$. We next give a broad generalization of Theorem 2.1. Its implication for Krull domains will be delayed until the next section.

THEOREM 2.3. Let $I$ be a finite type $v$-ideal of an integral domain $R$. Suppose that for each maximal ideal $M$ of $R$, there exists a positive integer $n_{M}$ (depending on $M$ ) such that $I_{M}^{\left(n_{M}\right)}$ is principal. Then there exists a positive integer $N$ such that $I^{(N)}$ is invertible.

Proof. Let $I=J_{v}$, where $J$ is finitely generated. Let $M \in \operatorname{Max}(R)$. Then $I_{M}^{\left(n_{M}\right)}=a R_{M}$ for some $a \in I^{\left(n_{M}\right)}$. Thus

$$
R_{M}=\left(a R_{M}: I_{M}^{\left(n_{M}\right)}\right) \subseteq\left(a R_{M}: J_{M}^{n_{M}}\right)=\left(a R: J^{n_{M}}\right)_{M}=\left(a R: I^{\left(n_{M}\right)}\right)_{M} .
$$

Hence there exists an $f_{M} \in R-M$ with $f_{M} I^{\left(n_{M}\right)} \subseteq a R$. Thus $f_{M}^{k} I^{\left(n_{M}\right) k} \subseteq a^{k} R$, so that $f_{M}^{k} I^{\left(n_{M} k\right)} \subseteq a^{k} R$ for all $k \geq 1$. It follows that $I^{\left(n_{M} k\right)} R_{f_{M}}=\overline{a^{k}} R_{f_{M}}$. Since $R=\left(\left\{f_{M} \mid M \in \operatorname{Max}(R)\right\}\right)$, we have that $R=\left(f_{1}, \ldots, f_{m}\right)$ for some finite set of maximal ideals $\left\{M_{1}, \ldots, M_{m}\right\}$ with $f_{M_{i}}=f_{i} \in R-M_{i}$. Put $N=n_{M_{1}} \cdots n_{M_{m}}$. Then $I^{(N)} R_{f_{i}}=a_{i}^{N / n_{M_{i}}} R_{f_{i}}$, where $I_{M_{i}}^{\left(n_{M_{i}}\right)}=a_{i} R_{M_{i}}$. Since for each maximal ideal $M$ of $R$, we have some $f_{i} \notin M$, it follows that $I_{M}^{(N)}=I^{(N)}\left(R_{f_{i}}\right)_{M_{f_{i}}}$ is principal. By Theorem 2.1, $I^{(N)}$ is invertible. 
3. Krull domains. Let $R$ be a Krull domain with quotient field $K$. We adopt the following standard notation: $X^{(1)}(R)=X^{(1)}$ is the set of height-one prime ideals of $R, \operatorname{Pic}(R)$ is the Picard group of $R$, and $\mathrm{Cl}(R)$ is the divisor class group of $R$. The factorial domains form the class of Krull domains with $\mathrm{Cl}(R)=0$. Factorial domains are of course characterized by the property that every principal ideal is a product of principal prime ideals. An integral domain $R$ (not necessarily a Krull domain) is said to be locally factorial if $R_{M}$ is factorial for each $M \in$ $\operatorname{Max}(R)$. A locally factorial domain need not even be a Krull domain (the example given at the end of $\S 2$ is locally a DVR, but not a Dedekind domain). An integral domain in which every principal ideal is a product of prime ideals (necessarily invertible) is called a $\pi$-domain. $\pi$-domains are precisely the locally factorial Krull domains. Theorem 3.1 surveys most of the known conditions equivalent to being a locally factorial Krull domain. Theorem 3.1 is repeated here as an example of the relationship between global and local results for Krull domains. Stated simply, a result concerning the invertible ideals of a Krull domain holds if and only if the corresponding result holds locally. While this was widely known for Noetherian Krull domains, it was apparently not so well known for non-Noetherian domains. Finally, $R(X)$ is the ring $R[X]_{S}$, where $S$ is the multiplicatively closed set of polynomials whose coefficients generate $R$.

THEOREM 3.1. For an integral domain $R$ the following conditions are equivalent:

(1) $R$ is a $\pi$-domain, i.e., every principal ideal of $R$ is a product of prime ideals.

(2) Every invertible ideal of $R$ is a product of invertible prime ideals.

(3) Every nonzero prime ideal of $R$ contains an invertible prime ideal.

(4) $R$ is locally factorial and every height-one prime ideal is finitely generated.

(5) $R$ is a locally factorial Krull domain.

(6) $R$ is a Krull domain and every $P \in X^{(1)}$ is invertible.

(7) $R$ is a Krull domain in which every divisorial ideal is invertible.

(8) $R$ is a Krull domain with $\mathrm{Pic}(R)=\mathrm{Cl}(R)$.

(9) $R$ is a Krull domain and each product of divisorial ideals is divisorial.

(10) $R$ is a Krull domain and the intersection of any two (principal) invertible ideals is invertible.

(11) $R$ is a Krull domain and for each $P \in X^{(1)}$, there exists a positive integer $n$ (depending on $P$ ) such that $P^{(k)}$ is invertible for $k \geq n$.

(12) $R(X)$ is factorial.

PROOF. The proof of the equivalence of conditions (1) and (5)-(10) is given in [1]. The proof of the equivalence of conditions (1)-(6) and (12) may be found in [2]. It only remains to show that (11) is equivalent to the other eleven conditions. It is clear that (7) implies (11). Conversely, suppose that (11) holds and let $P \in$ $X^{(1)}$. Then there exists an $n>0$ with both $P^{(n)}$ and $P^{(n+1)}$ invertible. Then $P=P^{(n)^{-1}} P^{(n+1)}$ is invertible.

It is interesting to note that for a quasi-local domain $R$ (or more generally an integral domain $R$ with $\operatorname{Pic}(R)=0$ ) each of the conditions of Theorem 3.1 is equivalent to $R$ being factorial. Corollary 2.2 gives new proofs to several of the implications of Theorem 3.1. In fact, Corollary 2.2 is really "what makes Theorem 3.1 work". Several of these conditions had been known to be equivalent for Noetherian Krull domains $((5) \Leftrightarrow(1)$ in [8], (5) $\Leftrightarrow(7)$ in [5], for example). An excellent survey of locally factorial domains appears in [3]. 
Let $R$ be a Krull domain. One can define the group $G(R)=\mathrm{Cl}(R) / \operatorname{Pic}(R)[1, \mathbf{3}$, 4]. It follows from Theorem 3.1 that $R$ is locally factorial if and only if $G(R)=0$. Thus $G(R)$ measures the deviation of $R$ from being a $\pi$-domain (locally factorial Krull domain) in a manner analogous to $\mathrm{Cl}(R)$ measuring the deviation of $R$ from being a factorial domain. Functorial properties of $G(R)$ similar to those of $\mathrm{Cl}(R)$ are given in [4].

Storch [9] defined a Krull domain to be almost factorial if $\mathrm{Cl}(R)$ is a torsion group. The following theorem characterizes such rings [9 or 5 ].

THEOREM 3.2. For a Krull domain $R$, the following conditions are equivalent.

(1) $R$ is almost factorial, i.e., $\mathrm{Cl}(R)$ is torsion.

(2) Every subintersection of $R$ is a ring of quotients.

(3) Some power of each proper principal ideal is a product of principal primary ideals.

(4) For $f, g \in R$, there exists an integer $n$ (depending on $f$ and $g$ ) such that $R f^{n} \cap R g^{n}$ is principal.

(5) If $P \in X^{(1)}$, then $P^{(n)}$ is principal for some $n>0$.

(6) For $P \in X^{(1)}, P=\sqrt{(x)}$ for some $x \in R$.

Krull domains satisfying (6) have been called semifactorial in [7]. It is clear that $R$ is almost factorial if and only if the radical of each divisorial ideal is the radical of a principal ideal.

As in the case of locally factorial domains, a Krull domain may be locally almost factorial without being almost factorial (e.g., a Dedekind domain with nontorsion divisor class group). We define a Krull domain $R$ to be locally almost factorial if $R_{M}$ is almost factorial for each $M \in \operatorname{Max}(R)$. In analogy to Theorem 3.1 and Theorem 3.2 we may define a Krull domain $R$ to be almost locally factorial if $G(R)=\mathrm{Cl}(R) / \mathrm{Pic}(R)$ is a torsion group, or equivalently for each $P \in X^{(1)}$ there exists an $n>0$ with $P^{(n)}$ invertible. Theorem 3.3 shows that there is no linguistic difficulty; almost locally factorial Krull domains are the same thing as locally almost factorial Krull domains.

THEOREM 3.3. For a Krull domain $R$, the following conditions are equivalent.

(1) $R$ is an almost locally factorial Krull domain, i.e., $G(R)=\mathrm{Cl}(R) / \mathrm{Pic}(R)$ is torsion.

(2) Every subintersection of $R$ is an invertible generalized quotient ring of $R$.

(3) For each prime $P \in X^{(1)}, P^{(n)}$ is invertible for some $n \geq 0$ (depending on $P$ ).

(4) Some power of every invertible ideal is a product of invertible primary ideals.

(5) For (principal) invertible ideals $I$ and $J$, there exists an integer $n>0$ for which $I^{n} \cap J^{n}$ is invertible.

(6) $R(X)$ is almost factorial.

(7) $R$ is locally almost factorial, i.e., for each $M \in \operatorname{Max}(R), \operatorname{Cl}\left(R_{M}\right)$ is a torsion group.

(8) Each $P \in X^{(1)}$ is the radical of an invertible ideal.

ProOF. The equivalence of (1)-(5) is given in [1]. In [4] it is shown that (1) and (6) are equivalent. Since the Picard group of a quasi-local domain is trivial, it is clear that $(3) \Rightarrow(7)$. The implication $(7) \Rightarrow(3)$ follows immediately from Theorem 2.3. It is clear that (3) implies (8). Conversely, suppose that (8) holds. Let $P \in X^{(1)}$ 
and let $P=\sqrt{I}$ where $I$ is invertible. But $I$ is then divisorial and $\sqrt{I}=P$, so we must have $I=P^{(n)}$ for some $n>0$. Thus (3) holds.

Condition (8) is easily seen to be equivalent to the radical of every divisorial ideal being the radical of an invertible ideal. An almost locally factorial Krull domain $R$ is almost factorial if and only if $\operatorname{Pic}(R)$ is torsion. Noetherian Krull domains satisfying condition (8) of Theorem 3.3 were studied by Mezzetti and Spangher in [7]. They proved the equivalence of certain of these conditions in the Noetherian case. The equivalence of (1) and (7) for $R$ Noetherian is given in [4]. One part of Theorem 3.3 (really Theorem 2.3) is so useful, that we restate it as a corollary.

COROLlARY 3.4. Let $R$ be a Krull domain. If $\mathrm{Cl}\left(R_{M}\right)$ is torsion for each $M \in$ $\operatorname{Max}(R)$, then $\mathrm{Cl}(R) / \mathrm{Pic}(R)$ is torsion. In particular, if $\mathrm{Pic}(R)=0, \mathrm{Cl}(R)$ is torsion if (and only if) $\mathrm{Cl}\left(R_{M}\right)$ is torsion for each $M \in \operatorname{Max}(R)$.

ADDED IN PROOF. W. Heinzer (Pacific J. Math. 29 (1969), 145-149) has shown that a nonzero prime ideal in a Krull domain that is both minimal and maximal is invertible. This result also follows from Corollary 2.2 since such an ideal is clearly locally principal. More generally, if $A$ is an ideal in a Krull domain such that $\sqrt{A}$ is a finite intersection of maximal ideals, then $A_{v}$ is invertible.

\section{REFERENCES}

1. D. D. Anderson, $\pi$-domains, overrings, and divisorial ideals, Glasgow Math. J. 19 (1978), 199203.

2. D. D. Anderson and J. Matijevic, Graded $\pi$-rings, Canad. J. Math. 31 (1979), 449-457.

3. A. Bouvier, Survey of locally factorial Krull domains, Publ. Dept. Math. Lyon (to appear).

4. __ The local class-group of a Krull domain, Canad. J. Math. (to appear).

5. R. Fossum, The divisor class group of a Krull domain, Springer-Verlag, Berlin and New York, 1973.

6. R. Gilmer, Multiplicative ideal theory, Dekker, New York, 1972.

7. E. Mezzeti and W. Spangher, Semifactorialité locale et anneaux de fractions généralisés, Boll. Un. Mat. Ital. B(5) 17 (1980), 1321-1337.

8. P. Maroscia, A note on locally factorial Noetherian domains, Comm. Algebra 9 (1981), 491-497.

9. U. Storch, Fastfaktorielle Ringe, Schr. Math. Inst. Univ. Münster No. 36 (1967).

Department of MAThematics, The UnIVERsity of IoWA, IOWA City, IOWA 52242 